\title{
Bullying y cyberbullying escolar en niños y jóvenes adolescentes: un estudio de caso
}

\section{School bullying and cyberbullying in children and young adolescents: a case study}

\author{
Miriam C. Ordóñez-Ordóñez (iD), Katerine D. Prado Cabrera (iD) \\ Facultad de Psicología, Universidad de Cuenca, Cuenca, Ecuador. \\ Autor para correspondencia: miriam.ordonez@ucuenca.edu.ec \\ Fecha de recepción: 17 de mayo de 2019 - Fecha de aceptación: 2 de octubre de 2019
}

\section{RESUMEN}

El objetivo de la investigación fue identificar la incidencia de bullying y cyberbullying en 983 estudiantes de 10 a 15 años de edad, del sexto al décimo año de EGB, pertenecientes a cinco instituciones educativas de la ciudad de Cuenca, para lo cual se cruzaron las variables de bullying y cyberbullying con la edad, el sexo y nivel educativo de los estudiantes encuestados, visualizando la implicación de los protagonistas, la tipología y sus manifestaciones. La metodología utilizada fue cuantitativa, con alcance descriptivo relacional, haciendo uso de dos instrumentos de medición: la escala European Bullying Intervention Project Questionnaire (EBIPQ) y la escala European Cyberbullying Intervention Project Questionnaire (ECIPQ), que permitieron identificar la incidencia y características del bullying y cyberbullying. Se evidenció un $28.3 \%$ de estudiantes víctimas de acoso escolar, de ellos un $20.7 \%$ eran víctimas de bullying, un $4.3 \%$ cibervíctimas y un $3.4 \%$ víctimas tanto de bullying como de ciberbullying. En el bullying la principal tipología presente era la verbal directa, en tanto que en el cyberbullying su principal manifestación fueron los insultos electrónicos y la exclusión. Se visualizó un mayor nivel de implicación de los varones como víctimas y agresores victimizados, en cuanto a la edad, los estudiantes de 12 a 15 años pertenecientes al octavo, noveno y décimo año presentaron un mayor grado de implicación como ciberagresores victimizados. En conclusión, se puede evidenciar que existe una mayor presencia del acoso escolar tradicional y una menor incidencia del cyberbullying, no obstante, parecería presentarse una relación directa entre las dos modalidades de acoso escolar.

Palabras clave: Acoso escolar, cyberbullying, acosador, víctima, acosador-victimizado.

\begin{abstract}
The objective of the research was to identify the incidence of bullying and cyberbullying in 983 students at age 10 to 15 , from the sixth to the tenth grade of EGB, belonging to five educational institutions in the city of Cuenca, for which the variables of bullying and cyberbullying were crossed with age, sex and educational level of the surveyed students, visualizing the involvement of the protagonists, the typology and its manifestations. The methodology used was quantitative, with relational descriptive scope, making use of two measurement instruments: The European Bullying Intervention Project Questionnaire (EBIPQ) and the European Cyberbullying Intervention Project Questionnaire (ECIPQ), which allowed to identify the incidence and characteristics of bullying and cyberbullying. There was evidence of $28.3 \%$ of students' victim of bullying, of them $20.7 \%$ were victim of bullying, $4.3 \%$ cyber victim and $3.4 \%$ victim of both bullying and cyberbullying. In bullying the main typology was direct verbal, while in cyberbullying its main manifestation were electronic insults and exclusion. A higher level of involvement of males was seen as victims and victimized aggressors. In terms of age, the 12- to 15-year-old students belonging respectively to the eighth, ninth and tenth grade presented a higher level of involvement as victimized cyber attackers. In conclusion, there is a greater presence of traditional school bullying and a lower incidence of cyberbullying, however, a direct relationship between the two forms of bullying seems to be present.
\end{abstract}

Keywords: Bullying, cyberbullying, stalker, victim, victimized stalker.

\section{INTRODUCCIÓN}

El acoso escolar es toda agresión física, verbal o psicológica de un niño o grupo de niños ejerce en contra de otro, acción que se repite y se mantiene en el tiempo. El acoso, como toda forma de violencia, afecta a los niños y adolescentes limitando un desarrollo psicológico saludable, un adecuado aprendizaje escolar y la inclusión en la sociedad. Es un fenómeno psicosocial multicausal, con una dinámica propia, cuya configuración involucra a tres actores (víctima, acosador, espectador), un contexto socioeducativo y al sistema educativo como tal. No es un fenómeno exclusivo del contexto educativo, es un reflejo de un dolor social (Kaplan, 2018), de un dolor que crece y se intensifica logrando instaurarse y plasmarse en el ámbito educativo y en su clima escolar. 
Las características que lo identifican son: la repetición y mantenimiento en el tiempo (agresión sistemática, deliberada y repetitiva) y la asimetría de poder (desequilibrio económico, social o físico) entre víctima y acosador. Se encuentra afianzado en dos leyes: a) la ley del silencio (víctima y espectador callan por temor a las represalias o porque lo sienten como un problema ajeno), y b) la ley de dominio-sumisión. En este entramado relacional la víctima se siente intimidada, excluida y débil, dado que las agresiones se intensifican progresivamente, las mismas que se presentan en su mayoría en privado (Mendoza, 2014). La víctima puede ser cualquier niño que presente características diversas al grupo y se deje afectar, no necesariamente "carece de habilidades sociales, con poca autoestima e introvertido ... cualquier rasgo diferenciador es susceptible de ser utilizado por quien acosa para convertir a otro niño en blanco de sus burlas" (Castro \& Reta, 2014, p. 54), en esta dinámica difusa el niño víctima en su intento de escapar del acoso puede terminar acosando como un desfogue catártico de su malestar.

El acoso escolar presenta una gran variedad de causas sociales, contextuales, personales (patrones conductuales de estrategias de afrontamiento) y familiares, a su vez consecuencias fundamentalmente para la víctima que abarcan desde un estado inicial de letargo, tristeza y flashbacks que van avanzando a un estado de ansiedad, distimia y depresión, acompañada de somatizaciones (dolor de cabeza, dolor de estómago, náuseas, vómitos y poliuria), no querer ir a la escuela, bajar su rendimiento escolar, tener pesadillas nocturnas, miedo, entre otros (Ordóñez, Mora, \& Pacheco, 2016; Mendoza, 2014).

Es un fenómeno complejo por su gran variedad de tipologías y manifestaciones, se presenta como acoso escolar tradicional (bullying) y también puede presentarse como cyberbullying. En lo referente al bullying tradicional, este puede ser: a) físico directo generado con la intención de producir daño o lesiones físicas, se manifiesta a través de: golpes, patadas, pellizcos, empujones y escupir, b) verbal directo que genera daño emocional en las víctimas, se presenta mediante insultos, rumores, chismes, c) verbal indirecto, a través de amenazas, d) daño a la propiedad del otro, por medio del robo o daño de las pertenencias y e) relacional que consiste en excluir socialmente a la víctima, su principal manifestación es el aislamiento (Ortega-Ruiz, 1997; Olweus, 1989; Mendoza, 2014).

El cyberbullying se caracteriza por el uso de instrumentos informáticos (internet, celular, redes sociales) con la finalidad de generar un daño constante y permanente, en donde el agresor mantiene el anonimato haciendo uso de un nombre falso para no ser identificado, colocando a la víctima en un mayor nivel de indefensión debido al avance masivo del acoso (Mendoza, 2014). Las manifestaciones del cyberbullying, a pesar de que guardan relación con el bullying tradicional, tienen características propias. Según Barlett (2019) existen por lo menos cuatro características propias que hacen que el cyberbullying se diferencie del bullying tradicional. En primer lugar, está la percepción de anonimato que se produce en el cyberbullying; por ejemplo, se pueden hacen rumores, burlas e insultos sin que la víctima sepa quién dio paso a tales comportamientos negativos. La forma más generalizada de acoso cibernético es que muchos usuarios de Internet pueden crear nombres de usuarios falsos, direcciones de correo electrónico únicas y cuentas de redes sociales falsas, para ocultar su verdadera identidad cuando están en línea. La segunda característica diferenciadora refiere a la asimetría de poder en el caso del cyberbullying; esta diferencia no necesariamente es física, debido a que no existe contacto entre el cyberagresor y la cybervíctima, más bien se ha demostrado que los agresores de esta modalidad de violencia reconocen que por medio de los dispositivos electrónicos su fisonomía es irrelevante. La tercera característica diferenciadora refiere a la repetitividad en el tiempo de las conductas agresivas. En el mundo virtual basta que se dé una publicación ofensiva contra otro, ésta podrá ser revisada y comentada por muchos otros. Finalmente, la cuarta característica hace referencia qué a diferencia del bullying tradicional, el cyberbullying no implica ningún tipo de contacto físico.

El cyberbullying se manifiesta a través de: a) insultos electrónicos/hostigamiento, haciendo uso de mensajes ofensivos, malintencionados y prosaicos, b) denigración, mediante la difusión de rumores inciertos que buscan dañar la reputación de la víctima, c) ciberpersecución, con el envío de mensajes amenazantes, d) suplantación de personalidad, haciéndose pasar por la víctima para enviar mensajes maliciosos, e) acoso cibernético por delegación /indirecto, haciendo uso de la cuenta de la víctima con la finalidad de enviar correos difamatorios a sus amigos, $\mathrm{f}$ ) desvelamiento, mediante la difusión de secretos o imágenes, y g) exclusión, generando una exclusión intencionada de la víctima a nivel de los grupos de redes sociales (Giménez, Arnaiz, \& Maquilon, 2013; Willard, 2005; Aftab, 2006; Mendoza, 2014).

El acoso escolar tradicional y el cyberbullying son dos modalidades de acoso, la primera directa (offline) y la segunda indirecta (online) dado que hace uso de la tecnología, pero no por ello menos grave. A nivel internacional las dos presentan una alta incidencia, evidenciable en varios estudios relacionados a la temática, en donde se visualiza la superposición entre el bullying y el cyberbullying, de manera específica en sus dos protagonistas: agresor y víctima, de tal manera que una víctima de acoso tradicional también puede ser una cibervíctima (Garaigordobil, Martínez-Valderrey, Páez, \& Cardozo, 2015; Castro \& Reta, 2014; Herrera-López, Romera, \& Ortega-Ruiz, 2017).

Se presentan una gran variedad de instrumentos que identifican la incidencia de bullying y cyberbullying, es así que, en el País Vasco, Machimbarrena \& Garaigordobil (2018) aplicaron la escala Cyberbullying: screening of peer-harassment para determinar la prevalencia de bullying y cyberbullying en estudiantes de primaria; en Murcia, González-Calatayud (2018) empleó el cuestionario Cyberbullying: screening of peer harassment, que le permitió identificar el porcentaje de cyberbullying, al igual que su tipología y manifestaciones. En su investigación Beltrán-Catalán, Zych, Ortega-Ruiz, \& Llorent (2018) describir en su estudio los diferentes patrones de victimización y la gravedad tanto de bullying como de cyberbullying, hicieron uso del European Bullying Intervention Project Questionnaire y el European Cyberbullying Intervention Project Questionnaire. Twardowska-Staszek, Zych, \& Ortega-Ruiz (2018) realizaron una investigación en Polonia donde validaron los instrumentos European Bullying Intervention Project Questionnaire y el European Cyberbullying Intervention Project Questionnaire. Analogo; Ortega-Ruiz, Del Rey, \& Casas (2016) validaron en España las escalas European Bullying Intervention Project Questionnaire (EBIPQ) y el European Cyberbullying Intervention Project Questionnaire (ECIPQ). Ellos obteniendo buenas 
propiedades psicométricas y evidenciando la relación entre el bullying y cyberbullying (lo que los convierte en instrumentos idóneos para evaluar de forma conjunta estos dos fenómenos), y destacan la influencia del bullying sobre el cyberbullying, evidenciando que una víctima de bullying también puede convertirse en víctima de cyberbullying. Finalmente, en Colombia Herrera-López, Romera, \& Ortega-Ruiz (2017) adaptaron y comprobaron las propiedades psicométricas de la escala European Bullying Intervention Project Questionnaire (EBIPQ), y encontrando óptimas propiedades psicométricas para los factores de victimización y agresión.

La escala European Bullying Intervention Project Questionnaire (EBIPQ) permite identificar la prevalencia de implicación en bullying, sus protagonistas: víctima, agresor y agresor victimizado, su tipología (directa e indirecta) y sus manifestaciones: físicas (golpear, patear, empujar), verbales (insultar, amenazar, decir palabras mal sonantes, difundir rumores), daño a la propiedad del otro (robar y romper las cosas) y relacionales (exclusión). La escala European Cyberbullying Intervention Project Questionnaire (ECIPQ) visualiza las dimensiones de cibervictimización y ciberagresión, evidenciando (por un periodo de tiempo de los dos últimos dos meses) sus manifestaciones a nivel de: insultos electrónicos / hostigamiento, denigración, ciberpersecusión, acoso cibernético por delegación/indirecto, suplantación de identidad, desvelamiento y exclusión (Ortega-Ruiz et al., 2016).

En base a estos antecedentes investigativos se abren una serie de interrogantes ligadas a ¿cuál es la incidencia de bullying y cyberbullying en nuestro contexto educativo?, en función de lo cual se ha planteado los siguientes objetivos de investigación:

- Determinar la incidencia de bullying y cyberbullying a nivel de víctimas, agresores y agresores victimizados;

- Identificar las manifestaciones de violencia desde la percepción de las víctimas y de los agresores; y

- Comparar la implicación en bullying y ciberbullying entre hombres y mujeres, entre niños de 10 a 11 años y jóvenes adolescentes entre 12 y 15 años, y entre los diferentes grados.

\section{MATERIALES Y MÉTODOS}

El presente es un estudio de corte transversal, no experimental, con enfoque cuantitativo $y$ alcance descriptivo relacional, en el que participaron niños y adolescentes de Educación General Básica (EGB). Las variables incluidas en el estudio estuvieron conformadas por: a) características sociodemográficas como: sexo, edad, tipo de familia ${ }^{1}$ y año de EGB, b) los protagonistas de acoso escolar: víctimas, agresores y agresores victimizados, c) su modalidad a nivel de acoso escolar tradicional o cyberbullying, d) su tipología, y e) sus manifestaciones.

La población de estudio estuvo constituida por 983 niños y adolescentes, de entre 10 y 15 años de edad, estudiantes de sexto a décimo año de EGB pertenecientes a cinco Instituciones Educativas del casco urbano de la ciudad de Cuenca, 4 de ellas eran fiscales y una privada; respectivamente la Unidad Educativa "A" con 252 estudiantes (25.6\%), Unidad Educativa "B" con 220 (22.4\%), Unidad Educativa "C" con 286 (29.1\%), Unidad Educativa "D" con 114 (11.6\%), y la Unidad Educativa "E" con $111(11.3 \%)$. De los cuales el 42.9\% fueron mujeres $(n=422)$ y el $57 \%$ hombres $(n=561)$, de entre 10 y 15 años de edad. El $49.7 \%$ de estudiantes pertenecía a familias nucleares, el $23.6 \%$ a familias monoparentales maternas sin soporte ${ }^{2}$ y con soporte de origen ${ }^{3}$, el $15 \%$ a familias extendidas, el $4.5 \%$ a familias reconstituidas y el $1.7 \%$ a familias monoparentales paternas sin soporte y con soporte de origen (ver Tabla 1).

La recolección de información inició con la obtención de los permisos por parte de la Coordinación Zonal 6 de Educación y de las autoridades educativas, se coordinó con los directivos de cada institución y se solicitó el consentimiento informado debidamente firmado por los representantes de los estudiantes participantes, así como el asentimiento de los niños y adolescentes, cumpliendo las normas éticas en materia de investigación; luego se visitó el plantel para administrar el cuestionario. Se explicó a los estudiantes el propósito del estudio y se garantizó el carácter anónimo, confidencial y voluntario de su participación. La aplicación de los instrumentos se realizó de forma colectiva en el aula de clase, dos evaluadores por clase, el tiempo medio de cumplimentación de los cuestionarios fue de 30 minutos.

Los datos se recolectaron mediante encuestas auto aplicadas empleando dos escalas: la escala European Bullying Intervention Project Questionnaire (EBIPQ) versión en español (Ortega-Ruiz et al., 2016) y la escala European Cyberbullying Intervention Project Questionnaire (ECIPQ) versión en español (Ortega-Ruiz et al., 2016). El EBIPQ mide la incidencia de bullying, lo

Tabla 1. Características de la población de estudiantes encuestados.

\begin{tabular}{lrr}
\hline Edad & $\mathrm{n}$ & $\%$ \\
\hline Diez & 218 & 22.2 \\
Once & 265 & 27.0 \\
Doce & 215 & 21.9 \\
Trece & 145 & 14.8 \\
Catorce & 116 & 11.8 \\
Quince & 24 & 2.4 \\
\hline Total & 983 & 100
\end{tabular}

\begin{tabular}{lrr}
\hline Tipo familia & $\mathrm{n}$ & $\%$ \\
\hline Nuclear & 489 & 49.7 \\
Extendida & 147 & 15.0 \\
Monoparental materna & 148 & 15.1 \\
Monoparental paterna & 9 & 0.9 \\
Monoparental materna con soporte de origen & 84 & 8.5 \\
Monoparental paterna con soporte de origen & 8 & 0.8 \\
Reconstituida & 44 & 4.5 \\
Reconstituida y extendida & 28 & 2.8 \\
Otra & 26 & 2.6 \\
\hline
\end{tabular}

\footnotetext{
Dato que permitió caracterizar a la población de estudio.

2 La madre educa sola a su hijo sin ningún apoyo familiar (Minuchin \& Fishman, 1997).
}

3 La madre educa a su hijo con apoyo de su familia de origen:
madre, padre, hermanos, o hijos mayores (Minuchin \& Fishman, 1997). 
Tabla 2. Kolmogorov-Smirnov con corrección de significación de Lilliefors para testear la normalidad del EBIPQ y ECIPQ.

\begin{tabular}{|c|c|c|c|}
\hline \multirow{2}{*}{ Variable } & \multicolumn{3}{|c|}{ Kolmogorov-Smirnov } \\
\hline & Estadístico $^{4}$ & $\mathrm{Gl}^{5}$ & Sig. ${ }^{6}$ \\
\hline \multicolumn{4}{|l|}{$\overline{\text { European Bullying Intervention Project Questionnaire (EBIPQ) }}$} \\
\hline B1. Alguien me ha golpeado, me ha pateado o me ha empujado & .328 & 272 & .000 \\
\hline B2. Alguien me ha insultado & .181 & 272 & .000 \\
\hline $\begin{array}{l}\text { B3. Alguien les ha dicho a otras personas palabras sobre mí porque quieren } \\
\text { hacerme daño }\end{array}$ & .332 & 272 & .000 \\
\hline B4. Alguien me ha amenazado & .477 & 272 & .000 \\
\hline B5. Alguien me ha robado o ha dañado mis cosas & .343 & 272 & .000 \\
\hline B6. Otras personas me han dejado de lado & .357 & 272 & .000 \\
\hline B7. Alguien ha dicho a otras personas chismes sobre mí & .260 & 272 & .000 \\
\hline B8. He golpeado. pateado o empujado a alguien & .460 & 272 & .000 \\
\hline B9. He insultado o he dicho palabras a alguien porque quiero hacerle daño & .512 & 272 & .000 \\
\hline B10. He dicho a otras personas palabras sobre alguien porque quiero hacerle daño & .523 & 272 & .000 \\
\hline B11. He amenazado a alguien & .532 & 272 & .000 \\
\hline B12. He robado o dañado alguna cosa de alguien & .528 & 272 & .000 \\
\hline B13. He dejado de lado a alguien (he ignorado) & .463 & 272 & .000 \\
\hline B14. He dicho a otras personas chismes sobre alguien & .482 & 272 & .000 \\
\hline \multicolumn{4}{|l|}{$\overline{\text { European Cyberbullying Intervention Project Questionnaire (ECIPQ) }}$} \\
\hline $\begin{array}{l}\text { C1. Alguien me ha dicho palabras o me ha insultado usando el internet o mensajes } \\
\text { por celular. porque quieren hacerme daño. }\end{array}$ & .446 & 278 & .000 \\
\hline $\begin{array}{l}\text { C2. Alguien ha dicho a otras palabras sobre mí usando internet o mensajes por } \\
\text { celular. porque quieren hacerme daño. }\end{array}$ & .447 & 278 & .000 \\
\hline C3. Alguien me ha amenazado a través de mensajes en internet o celular. & .508 & 278 & .000 \\
\hline C4. Alguien ha jaqueado mi cuenta de correo y ha robado mi información & .496 & 278 & .000 \\
\hline $\begin{array}{l}\text { C5. Alguien ha jaqueado mi cuenta de correo o redes sociales y se ha hecho pasar } \\
\text { por mí }\end{array}$ & .504 & 278 & .000 \\
\hline $\begin{array}{l}\text { C6. Alguien ha creado una cuenta de correo o redes sociales falsas para hacerse } \\
\text { pasar por mi }\end{array}$ & .521 & 278 & .000 \\
\hline C7. Alguien ha publicado información personal sobre mí en el internet & .521 & 278 & .000 \\
\hline C8. Alguien ha publicado videos o fotos comprometedoras sobre mí en internet & .531 & 278 & .000 \\
\hline C9. Alguien ha modificado fotos mías que yo había publicado en internet & .529 & 278 & .000 \\
\hline $\begin{array}{l}\text { C10. He sido dejado de lado (excluido. ignorado) o bloqueado de una red social o } \\
\text { del chat }\end{array}$ & .416 & 278 & .000 \\
\hline C11. Alguien ha dicho chismes sobre mí por internet & .450 & 278 & .000 \\
\hline $\begin{array}{l}\text { C12. He dicho palabras a alguien o le he insultado usando mensajes por celular o } \\
\text { internet. porque quiero hacerle daño. }\end{array}$ & .538 & 278 & .000 \\
\hline $\begin{array}{l}\text { C13. He dicho palabras sobre alguien a otras personas a través de mensajes por } \\
\text { celular o internet. porque quiero hacerle daño. }\end{array}$ & .541 & 278 & .000 \\
\hline C14. He amenazado a alguien a través de mensajes por celular o internet & .528 & 278 & .000 \\
\hline $\begin{array}{l}\text { C15. He jaqueado la cuenta de correo o redes sociales de alguien y me he robado } \\
\text { su información personal }\end{array}$ & .536 & 278 & .000 \\
\hline $\begin{array}{l}\text { C16. He jaqueado la cuenta de correo o redes sociales de alguien y me he hecho } \\
\text { pasar por él o ella. }\end{array}$ & .533 & 278 & .000 \\
\hline $\begin{array}{l}\text { C17. He creado una cuenta de correo de redes sociales falsa para hacerme pasar } \\
\text { por otra persona. }\end{array}$ & .537 & 278 & .000 \\
\hline $\begin{array}{l}\text { C18. He publicado información personal de alguien en el internet. porque quiero } \\
\text { hacerle daño. }\end{array}$ & .520 & 278 & .000 \\
\hline C19. He publicado videos o fotos comprometedoras de alguien en internet & .534 & 278 & .000 \\
\hline C20. He modificado fotos o videos de alguien que estaban publicados en internet & .532 & 278 & .000 \\
\hline $\begin{array}{l}\text { C21. He dejado de lado (excluido. ignorado) o bloqueado a alguien en una red } \\
\text { social o chat }\end{array}$ & .467 & 278 & .000 \\
\hline C22. He dicho chismes sobre alguien en internet & .536 & 278 & .000 \\
\hline
\end{tabular}

\footnotetext{
${ }^{4}$ Estadístico: diferencia absoluta máxima entre la curca de frecuencia normal y observada

5 gl: grados de libertad

6 Sig.: una variable no se distribuye normalmente si Sig. $<.05$
} 
integran 14 ítems, con cinco opciones de respuesta desde 0 a 4 (tipo Likert), siendo $0=$ nunca, $1=$ una vez o dos veces, $2=$ una 0 dos veces al mes, $3=$ alrededor de una vez por semana, y $4=$ más de una vez a la semana. Del total de ítems, 7 corresponden a situaciones de victimización y $7 \mathrm{de}$ agresión. Los valores de consistencia interna (alpha de Cronbach) de la versión adaptada al contexto, revelaron un alto grado de fiabilidad: $\alpha=.769$ para los ítems de victimización y $\alpha=.759$ para los de agresor.

El ECIPQ mide la incidencia de ciberbullying, está compuesto por 22 ítems de respuestas tipo Likert con cinco opciones desde 0 a 4 , siendo $0=$ nunca, $1=$ una vez $o$ dos veces, $2=$ una o dos veces al mes, $3=$ alrededor de una vez por semana, y $4=$ más de una vez a la semana. Del total de ítems, 11 se refieren a la cibervictimización y 11 a la ciberagresión. La consistencia interna de la versión adaptada al contexto mostró una alta fiabilidad $\alpha=.840$ para los ítems de cibervictimización y $\alpha=.732$ para los de ciberagresión.

En la sección inicial de las escalas se incorporaron variables sociodemográficas como: sexo y edad. Para determinar los diferentes roles de implicación tanto en el bullying tradicional cuanto en el cyberbullying se siguieron los criterios establecidos por los autores de las escalas: Brighi et al. (2012a, 2012b) y Del Rey et al. (2015):

- Un estudiante responde el rol de víctima o cibervíctima cuando los puntajes de respuesta son iguales o superiores a 2 (una vez al mes) en cualquiera de los ítems de victimización, y con puntaje igual o menor que 1 (una o dos veces) en todos los ítems de agresión.

- Un estudiante responde el rol de agresor o ciberagresor cuando sus puntuaciones son iguales o superiores a 2 (una vez al mes) en cualquiera de los ítems de agresión, y con puntaje igual o menor que 1 (una o dos veces) en todos los ítems de victimización.

- Un estudiante responde el rol de agresor-victimizado y ciberagresor victimizado si obtuvieron puntuaciones iguales o superiores a 2 (una vez al mes) en al menos uno de los ítems de agresión y de victimización.

El análisis descriptivo de las variables de estudio integro frecuencias y porcentajes, para el establecimiento de los factores asociados se hizo uso de la prueba de hipótesis chi-cuadrado, en el programa SPSS v. 25. El nivel de significancia utilizado fue de .05 .

\section{RESULTADOS}

En primer lugar, para conocer cómo se comportan los datos del instrumento EBIPQ y ECIPQ se aplicó la prueba de Kolmogorov-Smirnov. Encontrándose valores en las puntuaciones globales con niveles de significación de .000 . Esto permite rechazar la hipótesis de normalidad de datos, lo que sugiere el uso de pruebas no paramétricas para los análisis inferenciales (ver Tabla 2).
A nivel de resultados se encontró que el 28.3\% ( $\mathrm{n}=278)$ de estudiantes de la muestra son víctimas de acoso escolar, el $20.7 \%$ ( $\mathrm{n}=203$ ) son víctimas de bullying exclusivamente, el $4.3 \%(n=42)$ son cybervíctimas y el $3.4 \%(n=33)$ son víctimas de bullying y cyberbullying.

\subsection{Resultados bullying}

Se encontró un $24.1 \%$ ( $\mathrm{n}=236)$ de víctimas de bullying, un $3.3 \%(n=32)$ de agresores y un $13.1 \% \quad(n=129)$ de agresores victimizados, con una implicación del $40.4 \%$. A nivel de la percepción de las víctimas de bullying la tipología con mayor incidencia es la verbal directa, seguida de la física, la relacional indirecta, el daño a la propiedad del otro, y en última instancia la verbal indirecta. En cuanto a la percepción de los agresores se evidencia que en primera instancia se presenta la relacional indirecta seguida de la física, la verbal directa, y el daño a la propiedad del otro; el acoso verbal indirecto (amenazas) a criterio del agresor no está presente. Tanto victimas como agresores coinciden en la presencia de las mismas tipologías de acoso escolar, en lo que difieren es en la tipología que presenta mayor incidencia. Para la víctima la de mayor incidencia es la verbal directa, y para el agresor es la relacional indirecta. Un nivel de coincidencia es la visualización de que el acoso verbal indirecto (amenazas) se encuentra en los mínimos porcentajes, evidenciándose una mínima tendencia a la amenaza y una mayor tendencia a la ejecución del acto como tal (ver Tabla 3).

A pesar de que se pudo observar una mayor incidencia de víctimas, agresores y agresores victimizados en los niños $\mathrm{y}$ adolescentes varones, solo en víctimas $\chi^{2}(1 . \mathrm{n}=982)=$ $8.58, p=0.003$ y agresores victimizados $\chi^{2}(1 . \mathrm{n}=979)=$ 11.123, $p=0.001$ fue la diferencia significativa. No existieron diferencias estadísticamente significativas entre víctimas, agresores y agresores victimizados con respecto a los grupos de edad. También, las diferencias encontradas en el número de los protagonistas en los diferentes grados no presentan diferencia estadísticamente significativa (ver Tabla 4).

\subsection{Resultados de cyberbullying}

Se encontró un $7.7 \%(\mathrm{n}=75)$ de cibervíctimas, un $2.8 \%$ $(\mathrm{n}=27)$ de ciberagresores, y un $4.9 \% \quad(\mathrm{n}=48)$ de ciberagresores victimizados, que corresponde a una implicación total de $15.3 \%$ ( $\mathrm{n}=150)$. Desde la percepción de la cibervíctima se observa con un mayor nivel de incidencia los insultos electrónicos ("Me han insultado") y la exclusión ("Me han excluido, ignorado o bloqueado"), seguidos de la denigración ("Han hablado sobre mí para hacerme daño", "han dicho chismes sobre mí"), y en un menor nivel de incidencia el desvelamiento ("alguien ha publicado videos o fotos comprometedoras sobre mí"). Desde la perspectiva del ciberagresor en primera instancia se encuentra la exclusión, seguido de los insultos electrónicos, la ciberpersecusión ("He amenazado a alguien"), la suplantación de personalidad ("He jaqueado y robado información"), el acoso cibernético por delegación ("He jaqueado y me he hecho pasar por otro"), y con menores porcentajes la denigración mediante la propagación de chismes (ver Tabla 5). 
Tabla 3. Manifestaciones de acoso escolar desde la perspectiva de la víctima y del agresor.

\begin{tabular}{lrr}
\hline Variable & $\mathrm{n}$ & $\%$ \\
\hline Ítems de victimización $(\mathrm{n}=236)$ & 169 & 71.5 \\
Alguien me ha insultado & 131 & 55.5 \\
Alguien ha dicho a otras personas chismes sobre mí & 100 & 42.4 \\
Alguien ha hablado sobre mí para hacerme daño & 100 & 42.4 \\
Alguien me ha golpeado. pateado o empujado & 92 & 38.9 \\
Otras personas me han dejado de lado & 91 & 38.6 \\
Alguien me ha robado o ha dañado mis cosas & 42 & 17.8 \\
Alguien me ha amenazado & & \\
\hline Ítems de agresión (n=32) & 18 & 56.3 \\
He dejado de lado a alguien (he ignorado) & 17 & 53.1 \\
He golpeado. pateado o empujado a alguien & 11 & 34.4 \\
He dicho a otras personas chismes sobre otro & 8 & 25.0 \\
He insultado & 4 & 12.5 \\
He robado o dañado alguna cosa de alguien & 2 & 6.3 \\
He dicho palabras sobre alguien para hacerle daño & 0 & 0.0 \\
He amenazado a alguien &
\end{tabular}

Tabla 4. Implicación en acoso escolar según sexo, edad y grado.

\begin{tabular}{llcccccc}
\hline \multirow{2}{*}{ Variable de comparación } & \multicolumn{2}{c}{ Víctimas } & \multicolumn{2}{c}{ Agresores } & \multicolumn{2}{c}{ Agresores victimizados } \\
\cline { 3 - 7 } & & $\mathrm{n}$ & $\%$ & $\mathrm{n}$ & $\%$ & $\mathrm{n}$ & $\%$ \\
\hline \multirow{2}{*}{ Sexo } & mujeres $(\mathrm{n}=422)$ & 82 & 19.4 & 12 & 2.9 & 38 & 9.0 \\
& hombres $(\mathrm{n}=561)$ & 154 & 27.5 & 20 & 3.6 & 91 & 16.3 \\
\hline \multirow{2}{*}{ Edad } & $10-11$ años $(\mathrm{n}=483)$ & 116 & 24.0 & 12 & 2.5 & 58 & 12.1 \\
& 12-15 años (n=500) & 120 & 24.0 & 20 & 4.0 & 71 & 14.2 \\
\hline \multirow{3}{*}{ Grado } & sexto $(\mathrm{n}=193)$ & 44 & 22.8 & 7 & 3.6 & 25 & 13.1 \\
& séptimo (n=257) & 55 & 21.4 & 6 & 2.3 & 34 & 13.2 \\
& octavo $(\mathrm{n}=255)$ & 65 & 25.5 & 7 & 2.8 & 35 & 13.7 \\
& 9no y 10mo (n=278) & 72 & 25.9 & 12 & 4.3 & 35 & 12.6 \\
\hline
\end{tabular}

Tabla 5. Manifestaciones de acoso escolar desde la perspectiva de la cibervíctima y del ciberagresor.

\begin{tabular}{lrr}
\hline Variable & $\mathrm{n}$ & $\%$ \\
\hline Ítems de cibervictimización (n=75) & 38 & 50.7 \\
Me han insultado & 38 & 50.7 \\
Me han excluido. ignorado o bloqueado & 36 & 48.0 \\
Han hablado sobre mí para hacerme daño & 31 & 41.3 \\
Han dicho chismes sobre mí en internet & 26 & 34.7 \\
Me han jaqueado y robado mi información & 19 & 25.3 \\
Me han amenazado & 17 & 22.7 \\
Me han jaqueado y se han hecho pasar por mi & 13 & 17.3 \\
Han publicado información personal sobre mi & 12 & 16.0 \\
Cuenta falsa y se han hecho pasar por mi & 8 & 10.7 \\
Han modificado mis fotos & 8 & 10.7 \\
Me han publicado videos o fotos comprometedoras & & \\
\hline Items de ciberagresión (n=27) & 17 & 63.0 \\
He excluido. ignorado o bloqueado a alguien & 7 & 25.9 \\
He insultado & 7 & 25.9 \\
He amenazado a alguien & 6 & 22.2 \\
He jaqueado y robado información & 6 & 22.2 \\
He jaqueado y me he hecho pasar por otro & 6 & 22.2 \\
He dicho chismes sobre alguien & 5 & 18.5 \\
Cuenta falsa y me he hecho pasar por otro & 4 & 14.8 \\
He hablado sobre alguien para hacerle daño & 2 & 7.4 \\
He publicado información de otro para dañarlo & 2 & 7.4 \\
He publicado videos o fotos comprometedoras de alguien & 2 & 7.4 \\
He modificado fotos de alguien & &
\end{tabular}


Tabla 6. Implicación en ciberacoso escolar según el sexo, edad y grado.

\begin{tabular}{llcccccc}
\hline \multirow{2}{*}{ Variable de comparación } & \multicolumn{2}{c}{ Cibervíctimas } & \multicolumn{2}{c}{ Ciberagresores } & \multicolumn{2}{c}{ Ciberagresor victimizado } \\
\cline { 3 - 7 } & & $\mathrm{n}$ & $\%$ & $\mathrm{n}$ & $\%$ & $\mathrm{n}$ & $\%$ \\
\hline \multirow{2}{*}{ Sexo } & mujeres $(\mathrm{n}=422)$ & 25 & 5.9 & 10 & 2.4 & 12 & 2.8 \\
& hombres $(\mathrm{n}=560)$ & 50 & 9.0 & 17 & 3.1 & 36 & 6.4 \\
\hline \multirow{2}{*}{ Edad } & 10 - 11años $(\mathrm{n}=483)$ & 29 & 6.0 & 12 & 2.5 & 15 & 3.1 \\
& 12-15 años $(\mathrm{n}=500)$ & 46 & 9.2 & 15 & 3.0 & 33 & 6.6 \\
\hline \multirow{3}{*}{ Grado } & sexto $(\mathrm{n}=193)$ & 6 & 3.2 & 5 & 2.6 & 4 & 2.1 \\
& séptimo $(\mathrm{n}=257)$ & 19 & 7.4 & 6 & 2.3 & 12 & 4.7 \\
& octavo $(\mathrm{n}=255)$ & 21 & 8.2 & 5 & 2.0 & 18 & 7.1 \\
& 9no y 10mo $(\mathrm{n}=278)$ & 29 & 10.5 & 11 & 4.0 & 14 & 5.1 \\
\hline
\end{tabular}

A pesar de las aparentes diferencias, no se puede afirmar que son significativas en relación con el número de hombres y mujeres cibervíctimas y ciberagresores; sin embargo, si se presentó diferencia estadísticamente significativa en ciberagresores victimizados $\chi^{2}(1 . n=982)$ $=6.6527, p=0.0099$, siendo más frecuente en hombres. Con respecto a los grupos de edad sucede una situación similar. Existió diferencia con respecto a la edad de los ciberagresores victimizados $\chi^{2}(2 . \mathrm{n}=983)=6.459$, $p=0.011$, siendo más frecuente en los estudiantes de entre 12 y 15 años. En relación con los grados, se presentaron diferencias en cibervíctimas $\chi^{2}(3 . \mathrm{n}=983)=8.8472$, $p<0.0313$; mas no en ciberagresores y ciberagresores victimizados (ver Tabla 6).

\section{DISCUSIÓN}

El primer objetivo pretendió determinar la incidencia de bullying y cyberbullying a nivel de víctimas. agresores y agresores victimizados.

Los resultados muestran una presencia mayor de acoso escolar tradicional y un menor nivel de incidencia de cyberbullying. Un dato interesante es la presencia, aunque en porcentajes bajos, de niños y adolescentes víctimas tanto de bullying como de cyberbullying. No obstante, es pertinente destacar que en el grupo de estudio la incidencia del acoso escolar tradicional es mayor que el cyberbullying. A nivel del porcentaje de acoso escolar, este se encuentra dentro de los valores medios reportados para Latinoamérica, en tanto que el cyberbullying se encuentra dentro de los valores bajos en comparación a los reportados para Latinoamérica, Estados Unidos y Europa. Esta incidencia y relación es evidenciable en varios estudios; entre ellos el de Herrera-López et al. (2018) en su estudio bibliométrico sobre bullying y cyberbullying en Latinoamérica, visualizaron que numerosos estudios reportaron una prevalencia de bullying entre el 20 y el 30 por ciento (similar a lo reportado en EE.UU. y Europa). Con respecta al cyberbullying, se presentaba mayor diferencia en el rango; los valores oscilaban entre 2.5 y $42.5 \%$, rangos que también van acordes a lo reportado en EE.UU. y Europa. Al comparar ambas prevalencias, del bullying y del cyberbullying, los autores sugieren por un lado la posibilidad de que existan dinámicas similares en el desarrollo y la ocurrencia de ambos fenómenos sin notables diferencias a pesar de las distancias culturales, idiomáticas y geográficas; y por otro. en el caso del cyberbullying que posiblemente la brecha tecnológica entre países en vía de desarrollo y los desarrollados sea cada vez menor.

Muijs (2017) en un estudio realizado en Reino Unido reportó que el $21 \%$ de la muestra estudiada era víctima de bullying, mientras que el $11 \%$ informaba haber sido perpetuadores de esta situación. García-Fernández, Romera-Félix, \& Ortega-Ruiz (2016) consideran en función de los resultados de su investigación que el estar involucrados en problemas de cyberbullying parecería ser un factor relacionado con la implicación en problemas de acoso tradicional, dado que los agresores de cyberbullying tendían a ser víctimas en bullying tradicional y las víctimas a través de vía electrónica se catalogaban como agresoras y agresoras victimizadas de bullying tradicional. Posiblemente, entre las razones por las que se justificarían estas relaciones en las que los ciberagresores puedan ser víctimas de bullying tradicional, los autores adoptan la concepción de Ybarra \& Mitchell (2004), quienes señalan que las Tecnologías de la Información y Comunicación (TIC) cumplen un rol compensatorio de aquello que no pueden hacer cara a cara. En cuanto a las relaciones negativas entre estas dos variables, se distingue cómo el grupo de los no implicados en cyberbullying tampoco suelen estarlo en el bullying tradicional y viceversa. Herrera-López et al. (2017) encontraron que la agresión en bullying influyó de manera directa sobre la implicación en ciberagresión; de igual manera, la victimización incidió sobre la implicación en cibervictimización. Los autores sugieren que la participación en cyberbullying se ve influenciada parcialmente por la implicación en bullying tradicional, pero no en dirección opuesta; es decir, que si un adolescente está implicado en conductas de agresión a otros de forma tradicional (bullying), tiene más probabilidades de implicarse en ciberagresión, pero también de convertirse en cibervíctima.

En su investigación realizada en España donde participaron 346 sujetos de entre 12 y 18 años, Nacimiento \& Mora-Merchán (2014) evidenciaron que el 51.5\% de los participantes habían sufrido con baja frecuencia (un máximo de dos veces al mes) episodios de cibervictimización y el $47.2 \%$ episodios de bullying tradicional. Sin embargo, las víctimas habituales (al menos una vez a la semana) comprendían el $28.2 \%$ en cyberbullying y el $40.6 \%$ de bullying tradicional.

En cuanto a la participación de los protagonistas en el acoso tradicional (bullying), existe una mayor presencia de víctimas, seguidas de víctimas y atacantes; mientras que con respecto al cyberbullying hay una mayor implicación de cibervíctimas, seguida de ciberagresores 
victimizados y ciberagresores, que muestran una situación similar con el acoso escolar tradicional. El mayor nivel de implicación se presenta en las víctimas y agresores victimizados, no obstante, la implicación de las víctimas en el acoso escolar tradicional es mayor a la implicación de la cibervíctima; data que coincide con lo encontrado por varias investigaciones como la de Herrera-López et al. (2017). Esto autores realizado su estudio en la ciudad de Pasto, Colombia, con la participación de 1931 adolescentes que cursaban la educación secundaria. Estos autores concluyeron que la implicación total en este fenómeno fue del $41.9 \%$, en donde el $23.4 \%$ de los estudiantes eran víctimas, el $4.5 \%$ agresores y el $14 \%$ agresores victimizados.

García-Fernández et al. (2016) identificaron que el 76\% de los estudiantes investigados mostraron una implicación en el fenómeno para todos los roles, siendo los más activos (víctimas. agresores y agresores victimizados) aquellos que presentaron índices de participación bajos; las víctimas mostraron un índice de $12.4 \%$, agresores de $19.9 \%$ y agresores victimizados de $15.4 \%$. Para el cyberbullying, los datos presentaron una menor frecuencia; concretamente, el $31.2 \%$ de los escolares se autonominó como implicado en alguno de los diferentes roles. Los datos evidenciaron que el $9.3 \%$ había sido víctima, un $5.5 \%$ agresores y un $3.4 \%$ agresores victimizados.

El segundo objetivo consistía en identificar las manifestaciones de violencia desde la percepción de las víctimas y de los agresores.

Se evidencia una coincidencia desde la perspectiva de la cibervictima y ciberagresor referente a que la tipología de cyberbullying con mayor incidencia son los insultos electrónicos y la exclusión; la exclusión también presentaba un alto nivel de incidencia en el acoso escolar tradicional desde la perspectiva del agresor. Respecto al bullying tradicional se evidencia tanto desde la perspectiva de la víctima cuanto del agresor como principales manifestaciones de acoso la verbal directa y la física (Aftab, 2006; Giménez et al., 2013; Ortega-Ruiz, 1997; Olweus, 1989; Willard, 2005). Los resultados coincidentes con los de Acosta \& Cisneros (2019), quienes identificaron como principal tipología de acoso escolar la verbal directa a través de manifestaciones centradas en los insultos, apodos, chismes y hablar mal de alguien. En cuanto al cyberbullying, la data es similar a la presentada por Machimbarrena \& Garaigordobil (2018) quienes evidencian como principales manifestaciones de cibervictimización la recepción de mensajes ofensivos/insultantes, las llamadas anónimas para asustar, el chantaje y la difamación. En cuanto a la ciberagresión, identifican la presencia de un mayor porcentaje de envío de mensajes ofensivos/insultantes y la realización de llamadas anónimas con la finalidad de asustar. Parecería ser que el acoso verbal, manifestado en la presencia de insultos es un denominador común tanto en el bullying tradicional y el cyberbullying.

El tercero de los objetivos iba dirigido a comparar la implicación en bullying y ciberbullying entre hombres y mujeres, entre niños de 10 a 11 años y adolescentes entre 12 y 15 años, y entre los diferentes grados.
Los resultados ligados al sexo identifican que existe una mayor implicación de los niños y adolescentes varones en los roles de víctimas y agresores victimizados de bullying. A nivel de cyberbullying existe una mayor implicación de los ciberagresores victimizados. Por tanto, el rol de agresor victimizado tanto en bullying como en cyberbullying presentan un mayor nivel de implicación a nivel del sexo masculino, data coincidente con otros estudios a nivel de Latinoamérica. Es así que HerreraLópez et al. (2017) evidenciaron que los estudiantes varones son en mayor medida víctimas, agresores y agresores victimizados. Giménez et al. (2013) identifican un $9.8 \%$ de agresores con un ligero predominio de niños $(55.3 \%)$ en comparación a las niñas $(44.7 \%)$.

En cuanto a la edad no existen diferencias estadísticamente significativas a nivel de la implicación de los estudiantes del grupo de estudio en los roles de bullying. En lo referente a cyberbullying los ciberagresores victimizados de 12 a 15 años presentan un mayor nivel de implicación; data que corresponde con investigaciones a nivel de Latinoamérica, especialmente en Colombia en donde Herrera-López et al. (2017) encontraron asociación significativa en los agresores victimizados de 14 y 15 años. García-Fernández et al. (2016) coincide también que los estudiantes de 14 y 15 años presentan mayor implicación como cibervíctimas y ciberagresores.

En lo referente al grado de escolaridad no se presentan diferencias estadísticamente significativas en los roles de bullying de los estudiantes encuestados. No obstante, a nivel de cyberbullying se evidenció que los estudiantes de noveno, décimo y octavo año de EGB presentan un mayor nivel de implicación como cibervíctimas; resultado semejante al evidenciado por Herrera-López et al. (2017), quienes identificaron que los estudiantes de octavo de básica se implicaron más como agresores y como agresores victimizados.

Los hallazgos revisten importancia para el ámbito educativo y social, dado que pueden servir como línea base contextualizada para generar procesos de prevención e intervención psicoeducativa y psicosocial de este fenómeno.

Las limitaciones de este estudio son propias de su carácter transversal de análisis, que puede reducir la posibilidad de las inferencias relacionales, al igual que la interpretación de la direccionalidad de las influencias visualizadas. Se presentan además limitaciones ligadas al uso y autoaplicación de las encuestas.

\section{CONCLUSIONES}

En términos generales se evidencia que el $28.3 \%$ de la muestra estudiada ha sido víctima de acoso escolar, en su mayoría de la forma tradicional y en menor medida han sido víctimas de cyberbullying o de ambas, en cuanto a las manifestaciones desde la perspectiva de las víctimas. En el acoso escolar tradicional se evidenciaron primordialmente los insultos, mientras que en el ciberbullying se suma la violencia relacional (exclusión). Sin embargo, también se encontró presente en un 
porcentaje considerable la violencia verbal por medio de la propagación de chismes, rumores e insultos, y la violencia física. Tanto en víctimas de bullying como en los agresores victimizados, de bullying tradicional y ciberbullying, existen diferencias significativas entre hombres y mujeres, siendo en su mayoría varones. En cuanto a la relación con la edad no existen diferencias significativas ni en víctimas ni agresores, tanto en bullying tradicional como en ciberbullying; más si lo hay en ciberagresores victimizados, teniendo en su mayoría edades comprendidas entre los 12 y 15 años. Otra de las diferencias significativas que se encontró fue en cibervíctimas con respecto al año de educación básica al que pertenecen, siendo mayoritariamente de 9 no. 10mo y octavo.

\section{AGRADECIMIENTO}

Este estudio se realizó en el marco del proyecto «Estrategias de afrontamiento de los niños víctimas frente a situaciones de acoso escolar y cyberbullying», ganador del XV concurso de proyectos de investigación del Departamento de Investigación de la Universidad de Cuenca (DIUC) para quien va nuestro agradecimiento. De igual manera un agradecimiento muy especial a la Coordinación Zonal 6 de Educación y a las autoridades educativas de la Instituciones Educativas participantes que posibilitaron la ejecución de la presente investigación.

\section{BIBLIOGRAFÍA}

Acosta, P., \& Cisneros, J. (2019). El fantasma del acoso escolar en las unidades educativas. CienciAmérica: Revista de Divulgación Científica de la Universidad Tecnológica Indoamérica, 8(1), 74-89. doi: 10.33210/ca.v8i1.207

Aftab, P. (2006). Stop Cyberbullying: How cyberbullying works? Recuperado de http://www.stopcyberbullying.org/ how_it_works/direct_attacks.html

Barlett, C. P. (2019). Chapter 2 - Cyberbullying. Traditional bullying. and aggression: A complicated relationship. En Barlett, C. P. (Ed.). Predicting Cyberbullying (pp. 11-16). doi: 10.1016/B978-0-12816653-6.00002-9

Beltrán-Catalán, M., Zych, I., Ortega-Ruiz, R., \& Llorent, V. (2018). Victimisation through bullying and cyberbullying: Emotional intelligence. severity of victimisation and technology use in different types of victims. Psicothema. 30(2), 183-188. doi: 10.7334/psicothema2017.313

Brighi, A., Ortega, R., Pyzalski, J., Scheithauer, H., Smith, P. K., Tsormpatzoudis, H., ..., et al. (2012a). European Cyberbullying Intervention Project Questionnaire (ECIPQ) (Unpublished questionnaire). Recuperado de https://www/bullyingandcyber.net

Brighi, A., Ortega, R., Pyzalski, J., Scheithauer, H., Smith, P. K., Tsormpatzoudis, H., ..., Thompson, J. (2012b). European Bullying Intervention Project
Questionnaire (EBIPQ) (Unpublished manuscript). University of Bologna.

Castro, A., \& Reta, C. (2014). Bullying blando. bullying duro y ciberbullying: Nuevas violencias y consumos culturales. ( $1^{\mathrm{a}}$ ed., $1^{\mathrm{a}}$ reimp.) Rosario, Argentina: Homo Sapiens Ediciones.

Del Rey, R., Casas, J. A., Ortega-Ruiz, R., SchultzeKrumbholz, A., Scheithauer, H., Smith, P., ..., Plichta, P. (2015). Structural validation and cross-cultural robustness of the European Cyberbullying Intervention Project Questionnaire. Computers in Human Behavior, 50. 141-47. doi: 10.1016/j.chb.2015.03.065

Garaigordobil, M., Martínez-Valderrey, V., Páez, D., \& Cardozo, G. (2015). Bullying y cyberbulling: diferencias entre colegios públicos-privados y religiosos-laicos. Pensamiento Psicológico, 13(1), 3952.

García-Fernández, C., Romera-Félix, E., \& Ortega-Ruiz, R. (2016). Relations between Bullying and Cyberbullying: Prevalence and co-ocurrence. Pensamiento Psicológico, 14(1), 49-61. doi: 10.11144/Javerianacali.PPSI14-1.rbcp

Giménez, A., Arnaiz, P., \& Maquilon, J. (2013). Causas, medios y estrategias de afrontamiento en la agresión online en escolares de Murcia (España). Texto Livr: Linguagem e Tecnologia, 6(2), 2-18. doi: 10.17851/1983-3652.6.2.2-18

González-Calatayud, V. (2018). Victims of cyberbullying in the Region of Murcia: a growing reality. Journal of New Approaches in Educational Research, 7(1), 10-16. doi: 10.7821/naer.2018.1.245

Herrera-López, M., Romera, E. M., \& Ortega-Ruiz, R. (2018). Bullying y cyberbullying en Latinoamérica. Un estudio bibliométrico. Revista Mexicana de Investigación Educativa, 23(76), 125-155.

Herrera-López, M., Romera, E., \& Ortega-Ruiz, R. (2017). Bullying y cyberbullying en Colombia: Coocurrencia en adolescentes escolarizados. Revista Latinoamericana de Psicología, 49(3), 163-172. doi: 10.1016/j.rlp.2016.08.001

Kaplan, K. (2018). Perspectivas de la violencia escolar: Sociología de la educación. En Verdugo, M. (Presidencia). 1er Simposium - Taller: Violencia escolar. la expresión de una sociedad herida. Simposium llevado a cabo en la Facultad de Filosofía, Letras y Ciencias de la Educación de la Universidad de Cuenca, Cuenca, Ecuador.

Machimbarrena, J., \& Garaigordobil, M. (2018). Acoso y ciberacoso en educación primaria. Psicología Conductual, 26(2), 263-280.

Mendoza, B. (2014). Bullying: los múltiples rostros del acoso escolar. ( $2^{\mathrm{a}}$ ed.). México: Editorial Pax México.

Minuchin, S., \& Fishman. C. (1997). Técnicas de terapia familiar. (5 ${ }^{\text {a }}$ reimp.). Barcelona. España: Paidós Ibérica S.A.

Nacimiento, L., \& Mora-Merchán, A. (2014). El uso de estrategias de afrontamiento y habilidades metacognitivas ante situaciones de bullying y cyberbullying. European Journal of Education and Psychology, 7(2), 121-129. doi: 10.1989/ejep.v7i2.184 
Muijs, D. (2017). Can schools reduce bullying? The relationship between school characteristics and the prevalence of bullying behaviors. British Journal of Educational Psychology, 87(2), 255-272. doi: 10.1111/bjep. 12148

Olweus, D. (1989). Prevalence and incidence in the study of antisocial behavior: Definition and measurements. En Klein, M. (Ed.). Cross-National Research in SelfReported Crime and Delinquency (pp. 187-202). Dordrecht, The Netherlands: Kluwer Academic Publishers.

Ordóñez, M., Mora, J., \& Pacheco, M. (2016). Estudio descriptivo: Perfil psicológico del niño víctima de acoso escolar. Revista Médica HJCA, 8(2),108-116. doi: 10.14410/2016.8.2.ao.18

Ortega-Ruiz, R., Del Rey, R., \& Casas, J. (2016). Evaluar el bullying y el cyberbullying validación española del EBIP-Q y del ECIP-Q. Psicología Educativa, 22(1),71-79. doi: 10.1016/j.pse.2016.01.004.
Ortega-Ruiz, R. (1997). La convivencia escolar: qué es y cómo abordarla. Programa educativo de prevención de maltrato entre compañeros y compañeras. España. Consejería de Educación y Ciencia. Junta de Andalucía. Recuperado de http://creena.educacion.navarra.es/web/bvirtual/2018/0 6/01/la-convivencia-escolar-que-es-y-como-abordarla/

Twardowska-Staszek, E., Zych, I., \& Ortega-Ruiz, R. (2018). Bullying and cyberbullying in polish elementary and middle schools: Validation of questionnaires and nature of the phenomena. Children and Youth Services Review, 95, 217-225. doi: 10.1016/j.childyouth.2018.10.045

Ybarra, M., \& Mitchell, K. (2004). Youth engaging in online harassment: associations with caregiver-child relationships. Internet use. and personal characteristics. Journal of Adolescence, 27(3), 319-336. doi: 10.1016/j.adolescence.2004.03.007

Willard, N. (2005). Educator's guide to cyberbullying and cyberthreats. Center for Safe and Responsible Internet Use. 1-16. Recuperado de bcloud.marinschools.org/SafeSchools/Documents/BPCyberBandT.pdf 